\title{
LA CONSTRUCCIÓN DEL ESPACIO EN DOS SITIOS HISTÓRICOS DEL ÁREA DE VENTANIA, PROVINCIA DE BUENOS AIRES
}

\author{
María Cecilia Panizza ${ }^{1}$, María Gimena Devoto ${ }^{2}$ y Fernando Oliva ${ }^{3}$
}

Recibido: 1 de abril de 2019. Aceptado: 16 de agosto de 2019

\begin{abstract}
Resumen
Se propone un acercamiento al estudio de las evidencias materiales de ocupación del espacio a fines del siglo XIX, en dos sitios ubicados en el sector sur del Área Ecotonal Húmedo Seca Pampeana (AEHSP). El primero, San Carlos, se encuentra en el predio de la estancia Santa Ana en el partido de Coronel Suarez, en el sudoeste de la provincia de Buenos Aires. El otro sitio es la casa del primer intendente de la localidad de Puan, Rómulo Franco, localizada en la isla de Puan en el partido homónimo, próxima a la cabecera del distrito bonaerense. Ambos sitios presentan estructuras habitacionales remanentes, donde se evaluaron las distintas fases constructivas de las estructuras que se observan en la actualidad, con el fin de contribuir a determinar la funcionalidad del sitio. Para el análisis se consideraron las posturas teóricas de la arqueología de la arquitectura, las cuales implican estudiar la relación del ser humano con las construcciones que usa o elabora. Se intenta identificar cómo el medio es modificado y cómo los espacios construidos son diseñados con el objetivo de favorecer ciertas percepciones. Si se determina cómo los espacios son pensados y organizados para propiciar cierta percepción respecto a su entorno y a la construcción en sí misma, se puede acceder a la racionalidad del grupo que la creó y usó.
\end{abstract}

Palabras claves: arqueología histórica - arqueología de la arquitectura - siglo XIX - Ventania

\begin{abstract}
It proposes an approach to the study of material evidences of occupation of space by the end of the 19th century, in two sites located in the southern sector of the Área Ecotonal Húmedo Seca Pampeana (AEHSP). The first, San Carlos, is located in the grounds of the ranch Santa Ana in the party of Coronel Suárez, in the southwest of the province of Buenos Aires. The other site is the home of the first mayor of the town of Puan, Romulo Franco, located on the island of Puan in the municipality of the same name, next to the head of the Buenos Aires district. Both sites present residential structures remaining, where we evaluated the different construction phases of the structures that are currently observed, which can help determine the functionality of the site. The theoretical positions of the archaeology of architecture were considered for the analysis which involves studying the relationship of human
\end{abstract}

1 CONICET, Centro de Estudios Arqueológicos Regionales, Facultad de Humanidades y Artes, Universidad Nacional de Rosario. Entre Ríos 758, CP 2000 Rosario. mcpanizza@yahoo.com.ar

2 CONICET; Centro de Estudios Arqueológicos Regionales, Facultad de Humanidades y Artes, Universidad Nacional de Rosario. Entre Ríos 758, CP 2000 Rosario. gimenadevoto@outlook.com

${ }^{3}$ Centro de Estudios Arqueológicos Regionales \& Departamento de Arqueología, Facultad de Humanidades y Artes, Universidad Nacional de Rosario. Entre Ríos 758, CP 2000 Rosario. fwpoliva@gmail.com 
beings with constructions that uses or elaborates. Attempts to identify "how the medium is changed?" and "how the constructed spaces are designed with the aim of favouring certain perceptions?" If it is determined how the spaces are designed and organized to facilitate some perception with respect to its environment and the construction itself, you can access the rationality of the group who created it and used.

Keywords: historical archaeology - archaeology of architecture $-19^{\text {th }}$ century - Ventania

\section{Introducción}

Desde el año 2015 se desarrolla un proyecto de investigación acreditado en la Universidad Nacional de Rosario, centrado en el análisis del registro arqueológico histórico del área de Ventania (sierras australes bonaerenses), ubicada en el sector sur del Área Ecotonal Húmedo Seca Pampeana (AEHSP). A partir de estos estudios, se relevaron dos sitios con estructuras remanentes de fines del siglo XIX, uno ubicado al noreste del sistema serrano y el otro en el noroeste (Figura 1). El primero es el sitio San Carlos en el partido de Coronel Suárez y el segundo es la casa del primer intendente del partido de Puan, Rómulo Franco, quien en las décadas de 1880 y 1890 desempeñó varios cargos públicos, además del mencionado, fue juez de paz, presidente de la municipalidad, diputado, entre otros. Ambos sitios del período histórico han sido investigados en estos últimos años, la presentación de los mismos junto con los resultados de los análisis preliminares de su ubicación y de los materiales asociados han sido publicados recientemente (Oliva et al. 2016b, Panizza et al. 2016b, 2018).

En esta oportunidad se propone un acercamiento diferente para ambos sitios, mediante las herramientas provistas por la línea de investigación conocida como Arqueología de la Arquitectura. Las estructuras documentadas para ambos sitios son evaluadas en cuanto a sus parámetros constructivos, y se elabora la biografia de su construcción, funcionalidad y uso. Se lleva a cabo una aproximación al estudio arquitectónico de los sitios teniendo en cuenta su ubicación con respecto a otros sitios históricos cercanos, la cercanía a ciertos recursos como canteras, arroyos y bosques, la distancia a caminos y a las principales localidades, entre otros. Durante el trabajo de campo, se ha realizado la toma de medidas de las construcciones así como también la identificación de distintos momentos en la "vida" de las edificaciones donde se han utilizado distintas técnicas constructivas. Este abordaje se complementa con la lectura crítica de las fuentes documentales disponibles, la memoria oral de las comunidades locales, y los resultados de los relevamientos efectuados.

\section{Área de estudio y antecedentes de investigación}

Los sitios abordados en este trabajo se ubican dentro del área de Ventania, en el sector sur del Área Ecotonal Húmedo Seca Pampeana. El ecotono es un espacio de transición entre las regiones ecológicas de Pampa Húmeda y Seca, cuyos límites difusos han experimentado diversos procesos de avance y retracción a través del tiempo debido a los cambios ambientales ocurridos en distintos momentos del Holoceno (Oliva 2006; Oliva y Panizza 2015a). Específicamente el sector sur de este ecotono se caracteriza por la presencia del Sistema 


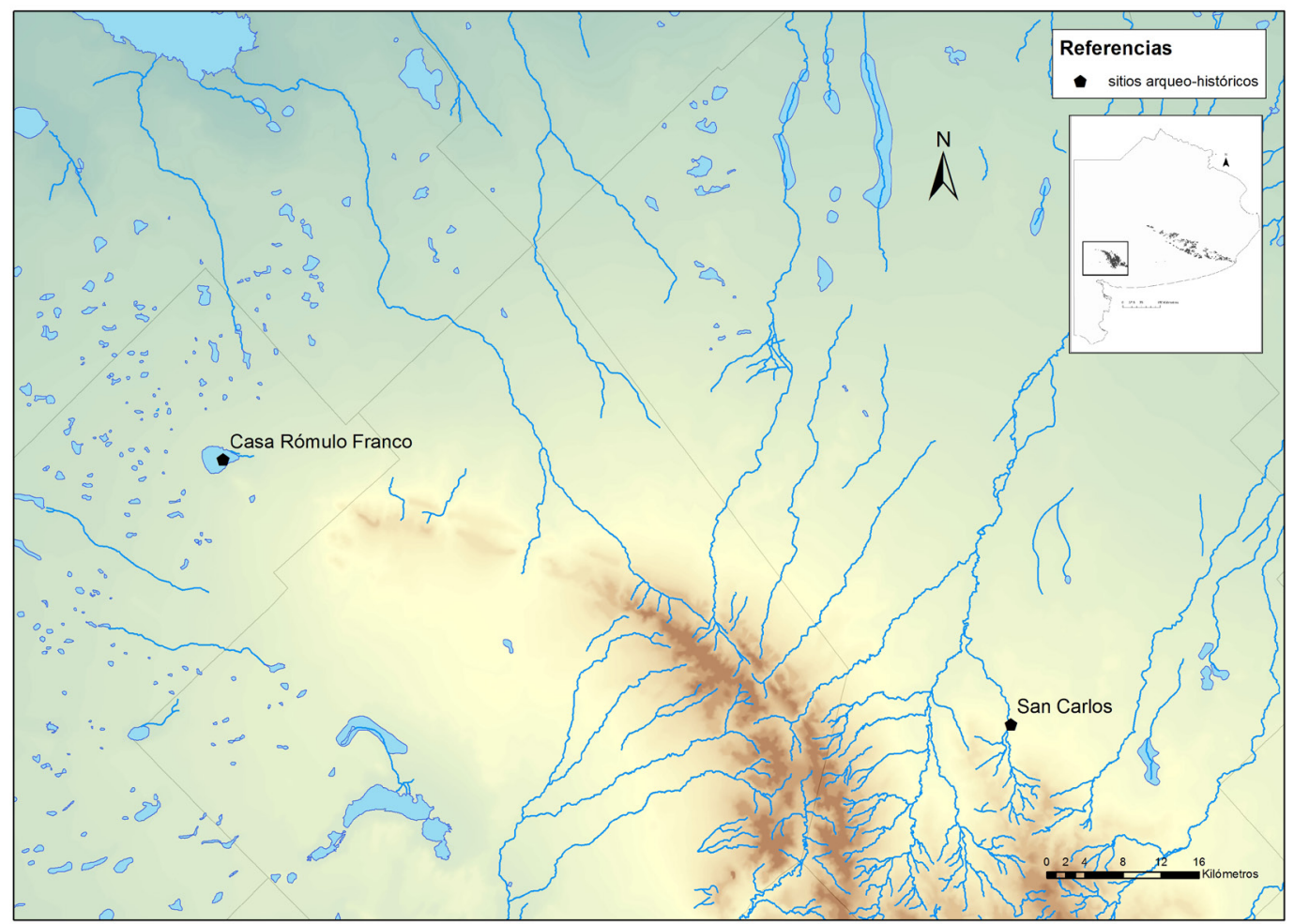

Figura 1. Mapa del sector norte del área de estudio (Ventania) con la ubicación de los dos sitios abordados en el presente trabajo.

Serrano de Ventania, que domina el paisaje de llanuras circundante, surcado por los numerosos cursos de agua que descienden de las sierras, como el arroyo El Perdido, algunos de los cuales alimentan lagunas de importantes dimensiones en la periferia, como la Laguna de Puan (Oliva et al. 2015a, 2015b).

La diversidad de ambientes y recursos de esta zona han resultado atractivos para que distintas poblaciones humanas hayan optado por habitarla los últimos 6000 años. A partir del siglo XVI comienzan una serie de procesos que aumentaron la complejidad de la trama social en la región, vinculados principalmente al ingreso de grupos de indígenas transcordilleranos y la posterior llegada de poblaciones de origen europeo. La ocupación y el contacto entre diversos grupos culturales desencadenaron profundos cambios sociales, políticos y económicos que se acentuaron en el siglo XIX (Devoto et al. 2016; Panizza 2015), relacionados a la formación y consolidación del Estado argentino.

Con respecto a los antecedentes de investigación sobre el registro arqueológico de momentos históricos del área de estudio propuesta, debe destacarse que son bastante recientes. A fines del siglo XX se inicia el abordaje de este tipo de sitios con los trabajos en el partido de Guaminí, al norte del sistema serrano de Ventania, donde se efectúan tareas en el Fortín Recompensa 
y el Fortín Barquín (Roa y Saghessi 1998). En el mismo partido se puede mencionar el sitio de superficie Jagüel en la margen norte de la Laguna del Monte donde se recuperaron diversos materiales de origen europeo (Petz y Saghessi 2007), entre los cuales pueden mencionarse fragmentos de vidrio de botellas de ginebra del siglo XIX, un fragmento de una pipa de caolín, una vaina servida de una bala, dos botones de metal de distinto tamaño, un objeto metálico no identificado, y dos hemiesferas de cobre. Otro hallazgo relevante se produjo en inmediaciones de la Laguna del Monte (partido de Guaminí), donde se registró el entierro de un individuo con deformación tabular erecta asociado a restos de un equino (Berón y Baffi 2003).

Otros enclaves militares investigados son el Fuerte San Martín y el Fortín Fe en el partido de Coronel Suárez (Langiano y Merlo 2013; Langiano et al. 2002a, 2002b; Merlo 2007) en el marco del estudio de los asentamientos en el Camino a Salinas. En los mismos, se analizó el uso de los recursos faunísticos en la dieta de los habitantes de puestos fortificados y la circulación de otros bienes materiales recuperados como cerámicas históricas, objetos de metal y de vidrio. En el Fuerte San Martín, específicamente, se investigaron las modificaciones en la distribución espacial de los elementos del componente arqueológico del basural y los procesos de formación de sitio, considerando variables como las reiteradas inundaciones en la zona y la deforestación de los sauces autóctonos como causas del stress vegetal que podría haber provocado cambios en las prácticas de estos grupos (Langiano et al. 2002b).

Por último, se ha trabajado un sitio correspondiente a un área formal de entierro del período de contacto indígena-criollo, Laguna Gascón 1, en un contexto con fauna introducida y materiales de tecnología indígena y exótica de alto valor simbólico, tales como frenos de caballo, cuentas de vidrio, hebillas y aros de metal, entre otros (Oliva y Lisboa 2006, 2009; Oliva et al. 2007). Recientemente, en el marco del proyecto mencionado en la introducción, parte del conjunto de información procedente de la evidencia material recuperada en este sitio ha sido re-evaluada, especialmente el material metálico (Oliva et al. 2015c) y las vasijas cerámicas (Oliva y Panizza 2017).

Actualmente en el área de Ventania se desarrollan líneas de investigación vinculadas a la cuestión de la frontera durante el siglo XIX a partir de fuentes etnohistóricas y del registro material (Devoto et al. 2016; Panizza 2015), el período de contacto hispano-indigena evidenciado en el arte rupestre (Oliva y Panizza 2015b, 2016), y se iniciaron las tareas arqueológicas sobre algunos de los fortines ubicados en la línea de frontera, la casa del primer intendente en el partido de Puan y el sitio San Carlos en el partido de Coronel Suárez (Oliva y Panizza 2018; Oliva et al. 2016; Panizza y Devoto 2018; Panizza et al. 2016;). Los dos últimos sitios mencionados son objeto de análisis en el presente trabajo debido a la existencia de estructuras de habitación, lo cual posibilita la aplicación de un estudio desde la perspectiva de la arqueología de la arquitectura.

\section{El área de estudio a fines del siglo XIX}

En el área en el que se inscribe la presente investigación, durante la segunda mitad del siglo XIX, se produjo la ruptura y búsqueda de la ocupación efectiva del territorio por parte del estado incipiente (que ya existía idealmente desde principios de siglo pero nunca se había concretado). Este avance ocasionó conflictos mayores, como las batallas de Pigüé (1858) y Curamalal (1877), ambas con la victoria oficial del ejército; y el corrimiento de la línea de frontera con el establecimiento de fuertes y fortines en el lapso 1862-1877, y la funda- 
ción de los primeros poblados (Monferran Monferran 1958, 1962; Thill y Puigdomenech 2003; entre otros).

La zona de las sierras fue un lugar de refugio para algunas de las parcialidades indígenas ante el conflicto, pero también un lugar atractivo para la ocupación por parte de las políticas estatales a partir de mediados del siglo XIX. Esta etapa vinculada con la actividad militar en la zona, culminó a fines del siglo XIX, con la realización de la primera conscripción argentina en el año 1896 en los campos de Curamalal, por lo cual se movilizaron aproximadamente ocho mil soldados a la localidad de Pigüé, que ubican su campamento en el Valle del Cerro Cura-Malal Grande (Baldrich 1896; Vigil 1959).

En el último cuarto del siglo XIX, el área de Ventania se empezó a delinear como una frontera cívica. Posteriormente a la "campaña al desierto", se produjo la ocupación sistemática y efectiva del territorio, junto con la instalación del ferrocarril. En esta etapa se fundaron los primeros poblados del área que dieron origen a las actuales localidades, como es el caso de Pigüé, Arroyo Corto, Saavedra, Tornquist, entre otros; el establecimiento de colonias de inmigrantes, entre las cuales pueden mencionarse las colonias de alemanes del Volga de Santa Trinidad, San José y Santa María, que se fusionaron en la localidad de Coronel Suárez; y el desarrollo de núcleos urbanos sobre los asentamientos militares previos, en Puan y en Saldungaray.

\section{Marco Teórico-metodológico: Arqueología de la Arquitectura}

La Arqueología de la Arquitectura empieza a surgir en Italia en las décadas de 1970 y 1980, pero el término recién se acuña en 1990 (Mannoni 1990), al mismo tiempo que en Inglaterra se inicia el estudio del edificio como objeto arqueológico (Utre- ro Agudo 2011), caracterizada por la incorporación de instrumentos específicamente estratigráficos (Azkarate et al. 2002); posteriormente se desarrollan distintas lineas, orientadas a la investigación y la restauración, como herramienta de análisis y de documentación, así como a la preservación de un patrimonio que, en algunos casos, está destinado a desaparecer.

En Latinoamérica los estudios de arqueología de la arquitectura se han referido a investigaciones tanto de época prehispánica, como colonial o contemporánea; se han ocupado de la arquitectura monumental o doméstica; la identificación de patrones de asentamiento, roles, funcionalidades, significados y/o articulación del paisaje, de contextos urbanos y de tecnologías de poder y disciplinamiento (Azkarate 2013).

La arquitectura y el paisaje son resultado de un proceso de apropiación del espacio y artificialización del medio, la arquitectura es una parte del paisaje, están relacionados porque la acción constructiva está en la génesis y la transformación de ambos (Blanco Rotea 2017). Se considera que son objetos del pasado en el presente susceptibles de ser estudiados desde distintas perspectivas para acceder a los mecanismos que los han producido; que son documentos del pasado que nos informan sobre distintos aspectos de las sociedades que los han generado; y que son recursos en el presente, que deben ser conservados y gestionados (Criado Boado 2012).

La arquitectura se comprende como una tecnología constructiva cuyo fin es brindar dimensión humana a un espacio (Mañana Borrazás et al. 2002), que se significa desde su propia estructura (el continente), los espacios que crea internamente (el contenido), las relaciones entre interior y exterior (Sennet 1991) o entre lo privado y lo público. En este sentido, también se consideran los avances de la Arqueología del Espacio Construido, con el objetivo 
de abordar de manera conjunta el estudio de la arquitectura como estructura (desde dentro de sí misma y en sí misma) fundamentalmente desde la Arqueología de la Arquitectura, y el análisis de la arquitectura en su relación con el entorno (hacia fuera de sí misma) desde la Arqueología del Paisaje (Blanco Rotea 2017).

Para abordar el análisis de los dos sitios estudiados, se consideraron las posturas teóricas mencionadas previamente, las cuales implican estudiar la relación del ser humano con las construcciones que usa o construye, intentando acceder así al tipo de racionalidad de un determinado grupo social. La arquitectura de un determinado sitio está dada por la manipulación antrópica de un espacio mediante técnicas constructivas que varian a lo largo del tiempo y que responden a factores sociales, culturales y económicos. La misma es resultado de una idea o percepción compartida por la colectividad de individuos de una sociedad (Mañana Borrazás et al. 2002). Los estudios enmarcados en dicha perspectiva teórica intentan identificar cómo el medio es modificado y cómo los espacios construidos son diseñados con el objetivo de favorecer ciertas percepciones. Si se determina cómo los espacios son pensados y organizados para propiciar cierta percepción respecto a su entorno y a la construcción en sí misma, se puede acceder a la racionalidad del grupo que la creó y usó (Criado Boado y Mañana Borrazás 2003). El análisis de la configuración formal de una construcción permite encontrar ciertas pautas de regularidad dentro de un patrón de racionalidad, e identificar recurrencias estructurales que indiquen la concepción del espacio para una determinada sociedad (Mañana Borrazás et al. 2002).

Con respecto a la metodología implementada, se propuso la evaluación de las distintas fases constructivas de las estructuras registradas en la actualidad, con el fin de establecer la funcionalidad del sitio.
Se ha llevado a cabo una aproximación al estudio arquitectónico de los sitios teniendo en cuenta su ubicación con respecto a otros sitios históricos cercanos, la cercanía a ciertos recursos como canteras, arroyos y bosques, la distancia a caminos y a las principales localidades, entre otros. Asimismo se ha realizado la toma de medidas de las construcciones así como también la identificación de distintos momentos en la "vida" de las edificaciones donde se han utilizado distintas técnicas constructivas. Por último, los espacios se clasificaron en abiertos, cerrados o construidos y de comunicación o circulación.

\section{Caso 1: sitio San Carlos (partido de Coronel Suárez)}

El sitio San Carlos está ubicado en el predio de la estancia Santa Ana en el partido de Coronel Suárez, a $20 \mathrm{~km}$ de la intersección entre las rutas 76 y 85, y próxima al sector noreste de las sierras de las Tunas (Sistema Serrano de Ventania, Provincia de Buenos Aires). Los primeros dueños registrados de la estancia Santa Ana fueron el matrimonio de Ana Cadmus y Tomas Hudson, oriundos de la ciudad de Nueva York, quienes construyeron la casona de estilo inglés en 1896, que en la actualidad se mantiene en buen estado de conservación. En 1905 la familia Amadeo y Videla compró la propiedad, y sus descendientes actualmente trabajan la estancia. A partir de relatos familiares, los propietarios refieren que en parte del casco se encontraba disimulado un fuerte, sin poder precisar el nombre del mismo, aunque llegaron a pensar en el Fuerte San Martín, pero investigaciones arqueológicas realizadas por Merlo y colaboradores (Langiano et al. 2002b) lo localizaron en una estancia cercana. Este sitio fue mencionado en un informe sobre el uso de la piedra en la construcción de fortines realizado por el 
Figura 2. A) Detalle de la Carta de la Provincia de Buenos Aires construida por el Departamento de Ingenieros con el registro gráfico de las propiedades rurales (1890). Se observa la parcela de Agustin Vidal (101), uno de sus extremos sobre el arroyo El Perdido. B) Detalle de la mensura $\mathrm{n}^{\circ} 101$ del partido de Coronel Suárez, correspondiente a Agustín Vidal en el año 1884, donde se observa un tramo del arroyo, próximo a la grafia "Est. a San Carlos" en el campo vecino de Gregorio Soler. C) Detalle de la mensura $n^{\circ} 38$ de Coronel Suárez (1880), donde se aprecia la señalización de tres cañones sobre los límites de la parcela, uno próximo al arroyo El Perdido. D) Detalle de la mensura $n^{\circ} 147 \mathrm{de}$ Coronel Suárez, donde se localiza "S $\mathrm{n}$ Carlos" a un costado del arroyo El Perdido y el antiguo camino, en la propiedad de Federico Cook (1896). E) Detalle del plano del partido de Coronel Suarez de 1924, editado por Lemann, único documento cartográfico donde aparece mencionado como Fortín San Carlos, ubicado frente al casco de la estancia Santa Ana. F) Detalle de la mensura ${ }^{\circ} 162$ de Coronel Suárez (1908), dentro del predio de la familia Videla Dorna se observan al oeste del arroyo El Perdido el casco de la estancia, al este el sitio San Carlos; y en el límite oriental de la parcela uno de los cañones mantiene su posición como mojón.

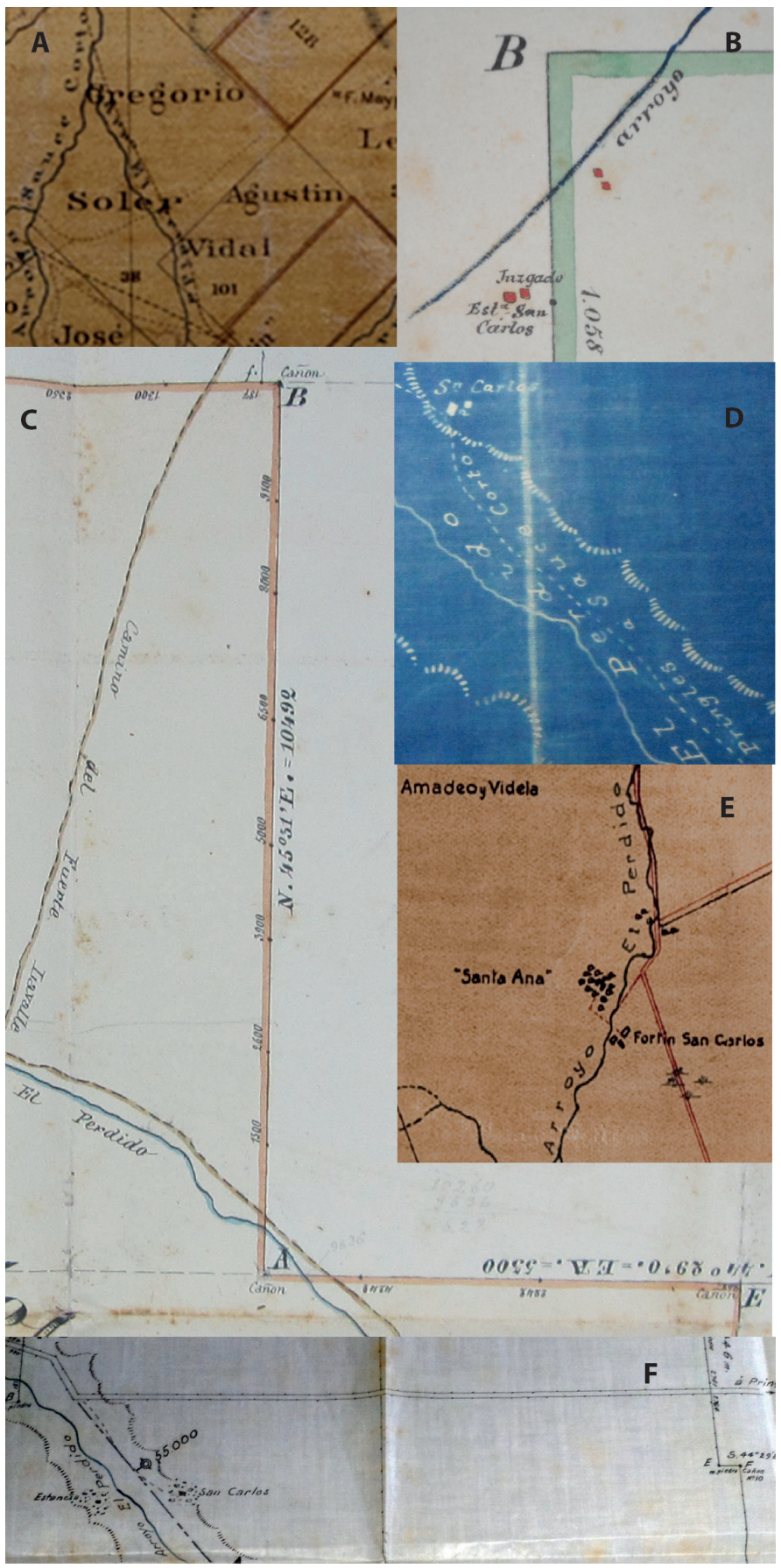


ingeniero Vila (2013), quien propone que sería un fortín de aprovisionamiento.

\section{San Carlos en el siglo XIX}

La búsqueda bibliográfica realizada en fuentes éditas e inéditas (Memorias de Guerra y Marina; Raone 1969; Thill y Puigdomenech 2003; entre otros) determinó la ausencia de referencia acerca de un fortín llamado San Carlos en la zona de estudio, pero el relevamiento de las fuentes cartográficas aportó un marco temporal para el surgimiento del asentamiento. Este marco establece que en las mensuras correspondientes a los años 1877 y $1880\left(n^{\circ} 1\right.$ y n $^{\circ} 38$ del partido de Coronel Suárez) todavía no figura el sitio San Carlos a orillas del arroyo El Perdido, en cambio se observan tres cañones como mojones en los lindes del campo (Figura 2C), uno de los cuales posteriormente fue trasladado al casco de la estancia, donde se encuentra actualmente. El sitio aparece con la denominación "Est. ${ }^{2}$ San Carlos" en la mensura del año 1884 (n ${ }^{\circ} 101$ de Coronel Suárez, Figura 2 A y B), y como "San Carlos", junto al arroyo "El Perdido" y al camino "de Pringles a Sauce Corto" en una mensura de 1896 ( $\mathrm{n}^{\circ} 147$ del municipio ya mencionado, Figura 2D), en otra mensura de 1908 ( $\mathrm{n}^{\circ}$ 162), donde también se representa la ubicación de un cañón y el casco de la estancia, coincidiendo con lo observado en la actualidad (Figura 2F). Los propietarios del establecimiento rural habrian tenido conocimiento de la existencia de dos cañones en los limites del campo, uno es el que estaría en "Santa Ana" y el otro en una estancia vecina (Rodolfo Serigós, comunicación personal). Finalmente, en el mapa catastral del partido de Coronel Suárez del año 1924 se señala la ubicación del Fortín San Carlos y la del casco de Santa Ana, emplazados en márgenes opuestas del Arroyo El Perdido (Figura 2E). Por otra parte, debe destacarse la existencia de restos de fortines en un área próxima al sitio, entre los cuales pueden mencionarse el Fuerte San Martín y el fortin 27 de diciembre.

\section{San Carlos en la actualidad}

El sitio San Carlos se orienta a $6^{\circ} \mathrm{N}$ sobre la margen derecha del Arroyo "El Perdido", un afluente del río Sauce Corto. Está constituido por un conjunto de estructuras, dentro del cual destaca la edificación principal con un patio interno y numerosas habitaciones, de construcción previa al casco de la estancia Santa Ana; varios corrales en sentido norte, un pozo de agua orientado al sur del sitio, y una pequeña habitación hexagonal en sentido este (Oliva et al. 2016). Esta última se encuentra separada del edificio principal, y habria cumplido la función de antigua carnicería rural desde principios de siglo $\mathrm{XX}$ hasta algunos años atrás: en el año 2016 fue refaccionada y convertida en una capilla (Figura 3A). Presenta techo también hexagonal de chapas, y aberturas de madera y vidrio en cuatro de las seis paredes: una puerta de dos hojas con dintel recto, tablero y paños vidriados (tres en cada hoja, seis en total); frente a la entrada una ventana rectangular con dos postigos internos, y en los laterales dos ventanas cuadradas con cuatro paños vidriados cada una y un postigo interno.

El pozo de agua con un brocal $^{1}$ de mampostería y dos postes de madera se ubica junto al lateral derecho de la edificación principal (Figura 3B); en cambio el antiguo camino del Sauce Corto a Pringles que figura en los mapas más antiguos de la zona, habría estado hacia el noroeste. Los corrales presentan muros realizados con rocas de carbonato cálcico chatas asentadas en barro (pircados), y luego revocada con mortero de cal (Figura 3C), estas piedras se obtuvieron del manto de carbonato característico de este sector de las sierras de Las Tunas. El revoque calizo posterior 


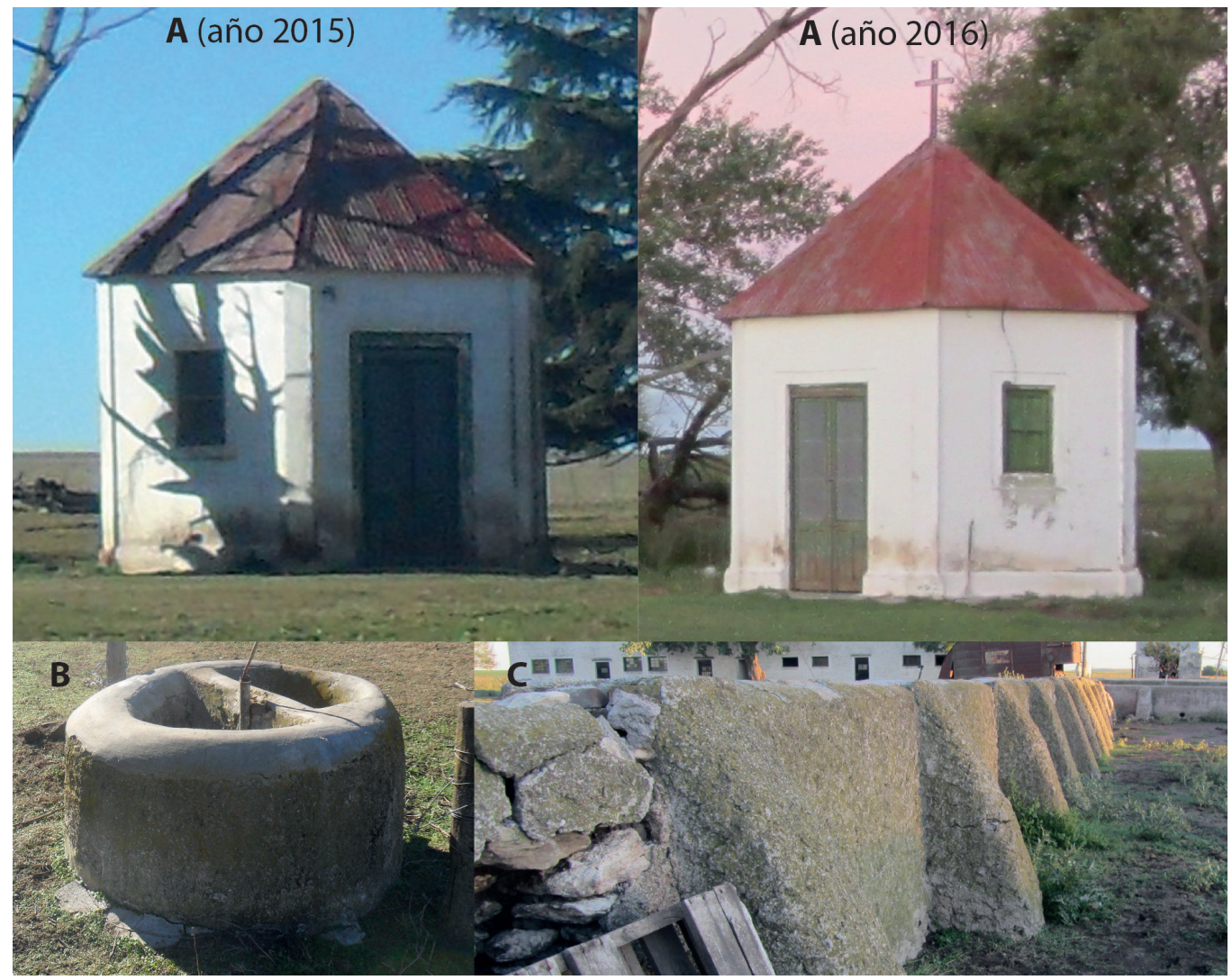

Figura 3. Estructuras anexas del sitio San Carlos. A) Antigua carniceria, hoy capilla. B) Pozo de agua. C) Corral con contrafuertes.

protege el pircado subyacente pero en algunos sectores se encuentra deteriorado; además presenta contrafuertes ${ }^{2}$ de contención como refuerzo de los extensos muros (68 x $40 \mathrm{~m})$.

Dentro del conjunto descripto se identificaron varias vinculaciones entre sí y con el entorno circundante. En primer lugar, es un sitio que posee una amplia visibilidad al frente sobre la llanura que lo rodea, y específicamente hacia las sierras de las Tunas al sudoeste, por encontrarse elevado unos metros sobre la superficie circundante. San Carlos y el casco de la estancia Santa Ana (del año 1896) están separados por $465 \mathrm{~m}$ hasta el corral y $535 \mathrm{~m}$ hasta la residencia. Esta disposición se correspondería con la organización espacial típica de las construcciones de los establecimientos rurales, con un agrupamiento en tres áreas (Moreno 1996): una sería la casa principal (Santa Ana en este caso) con un tratamiento arquitectónico más intenso, otra destinada al alojamiento del personal (San Carlos) correspondiente a los numerosos peones rurales necesarios para las tareas rurales de fines del siglo XIX, y por último un área donde se concentrarian los lugares de trabajo.

El Arroyo El Perdido, de curso perma- 
nente, se encuentra a $160 \mathrm{~m}$ de la estructura de corral y a $220 \mathrm{~m}$ de la edificación. La estructura de corral y el puesto fortificado se ubican a una distancia menor a $12 \mathrm{~m}$, entre este último y el recinto hexagonal hay $18 \mathrm{~m}$, y el antiguo camino entre Arroyo Corto y Pringles estaría a $85 \mathrm{~m}$ aproximadamente y el basurero próximo al sendero a $164 \mathrm{~m}$. La distancia a otros sitios arqueológicos históricos sería $8 \mathrm{~km}$ al oeste el Fuerte San Martín, entre los arroyos Sauce Corto y San Antonio; y más lejos hacia el oeste, a aproximadamente $18 \mathrm{~km}$, el fortín 27 de diciembre, sobre el arroyo homónimo.

\section{Análisis formal de la edificación principal}

En la edificación principal del sitio San Carlos, se utilizó ladrillo para construir los muros en la totalidad de las habitaciones. Los espacios se clasificaron en un espacio abierto (el patio central), tres espacios de circulación/comunicación, y 27 espacios cerrados (habitaciones de diferentes tamaños) (Figura 4). Es posible identificar una diversidad en la morfología de los ladrillos que habrian sido fabricados localmente, lo cual evidenciaría distintas fases cons- tructivas. La mayoria de las habitaciones presentan revoque en sus muros pero difieren en los componentes del mortero, por lo general se preparó con cal, arena y/o cemento pero hay habitaciones que fueron revocadas además con yeso, y otras donde el revoque es más poroso porque posiblemente contenía conchilla con cal. En la mayor parte de las habitaciones el techo es de madera y sólo en unas pocas es de chapa o ladrillo sobre vigas de madera. Los pisos de las mismas presentan en algunos casos baldosas o ladrillos y en otros casos sólo tierra. Los muros poseen dinteles y arcos de ladrillo para conformar las puertas $\mathrm{y}$ ventanas.

El frente de la construcción que se orienta al norte mide $35 \mathrm{~m}$, lo rodea una vereda de ladrillos y se observan 12 aberturas (tres puertas, una entrada abierta y ocho ventanas), todas con sus correspondientes guardapolvos ${ }^{3}$. De este a oeste se disponen: dos ventanas con 16 paños de vidrio cada una, una puerta de madera con ventanilla de vitraux enrejada verticalmente y tres bisagras de hierro con forma de flor de lis, dos ventanas similares a las anteriormente nombradas, una puerta de madera similar a la anterior pero con
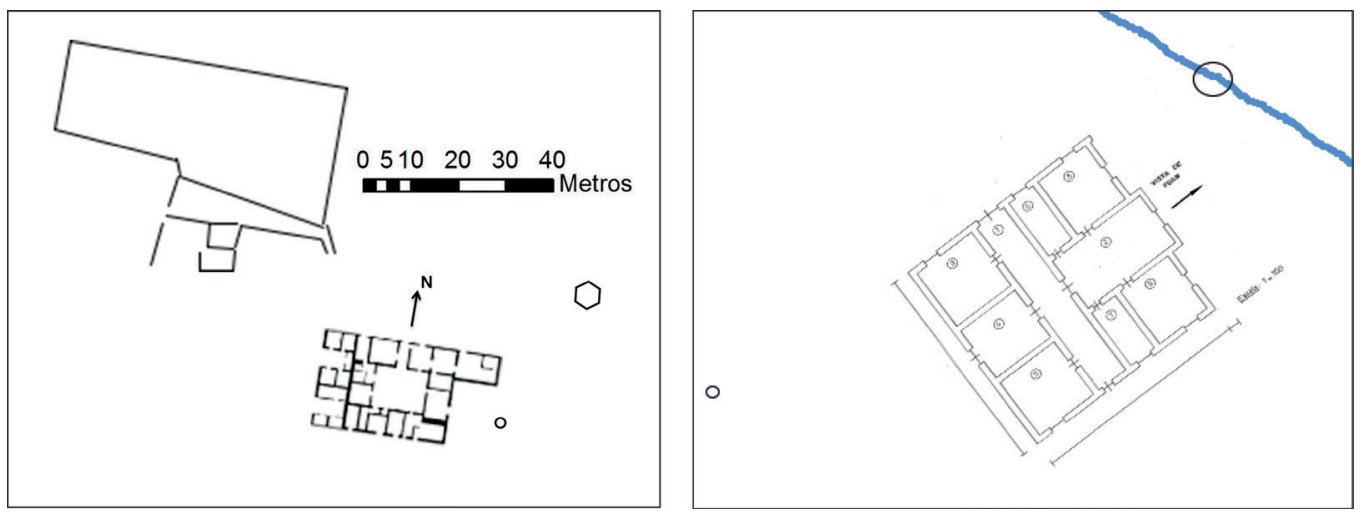

Figura 4. Planos donde se observa la disposición de las estructuras principales y asociadas para los dos casos analizados: San Carlos (izquierda) y Casa de Piedra de la Isla de Puan (derecha). 
cuatro paños de vidrio, la entrada abierta que comunica a un pasillo y éste al patio central (sobre esta abertura se ubica la espadaña ${ }^{4}$ de donde habría colgado la campana); dos ventanas rectangulares (a una le falta el vidrio, la otra presenta tres paños de vidrio), otra puerta parecida a las anteriores, con una ventanilla enrejada pero sin los herrajes; y dos ventanas rectangulares más (tres paños de vidrio cada una). Las puertas presentan grandes clavos ornamentados y rejas con balaustres torneados. Según la clasificación de Nadal Mora (1946), el frente corresponde a una fachada con espadaña central semicircular, el campanario se encuentra en la línea de la fachada. La espadaña central es el ápice natural de la fachada, que acentúa el sentido vertical y constituye un motivo que atrae las miradas por su situación elevada. Se clasifican según el número y disposición de aberturas (Nadal Mora 1946), en este caso está en una fila de una abertura, la cual es de arco semicircular, la cúpula es lisa.

El lateral este presenta forma de L, de norte a sur las ocho aberturas se organizan del siguiente modo: cuatro ventanas cuadradas con 16 paños de vidrio cada una, una ventana rectangular más pequeña, una puerta de metal con dos hojas, cada una con tres paños de vidrio (seis en total), una ventana de dos hojas con cuatro paños de vidrio en total y una puerta de madera.

El sector del frente norte y lateral este es el sector que presenta las mayores modificaciones y el que se encuentra más modernizado. Este sector fue habitado hasta tiempos más recientes, por lo tanto presenta los servicios de agua corriente, gas y electricidad habilitados. Este sector correspondia a las oficinas administrativas, comedores y un baño. Según los relatos orales habrian vivido en un primer momento parte de los integrantes de la familia dueña del campo y luego los capataces y su familia. Las habitaciones que conectan por el patio en el lado oeste presentan menos modificaciones, excepto aquella donde se ubica el sótano (Figura 5 A), ya que una pared de cartón yeso colocada sobre la trampilla de entrada a este espacio subterráneo la inutiliza y oculta. Además de este añadido que divide la habitación en dos cuartos se observa otra compartimentalización utilizada para instalar un baño.

El sector del contrafrente es el que presenta el mayor grado de deterioro, en algunos lugares falta el techo. Actualmente se encuentra en desuso, a excepción de una habitación que se utiliza como depósito. Algunos muros también están derrumbados y hay depositación de sedimentos en el suelo de las habitaciones. La mayoría de las habitaciones no conservaban sus aberturas. Se pudieron visualizar ventanas y puertas tapiadas. Las aberturas se disponen de la siguiente manera de este a oeste: una ventana de madera con mosquitero, una puerta de madera con ventani1la, una pequeña ventana cuadrada (sólo queda el agujero), la abertura correspondiente a una pequeña puerta de la que sólo subsiste el dintel de madera, otra abertura de una puerta de mayores dimensiones de la que se observa dintel y jambas, una pequeña ventana rectangular subdividida en dos franjas horizontales, otra pequeña ventana a mayor altura y protegida por un alero, una abertura con dintel de madera de la que cuelga una puerta de madera desvencijada, y dos ventanas con marco de madera, dintel de ladrillo en arco, no conservan los vidrios pero una de ellas todavía posee una contraventana de madera (Figura 5 C y D).

El patio central comunica directamente con ocho espacios de diferentes dimensiones correspondientes a los diferentes sectores (uno de los cuales es el pasillo que comunica con el exterior), excepto el lateral occidental (Figura 4). De estas aberturas solamente cuatro conservan las puertas, al 

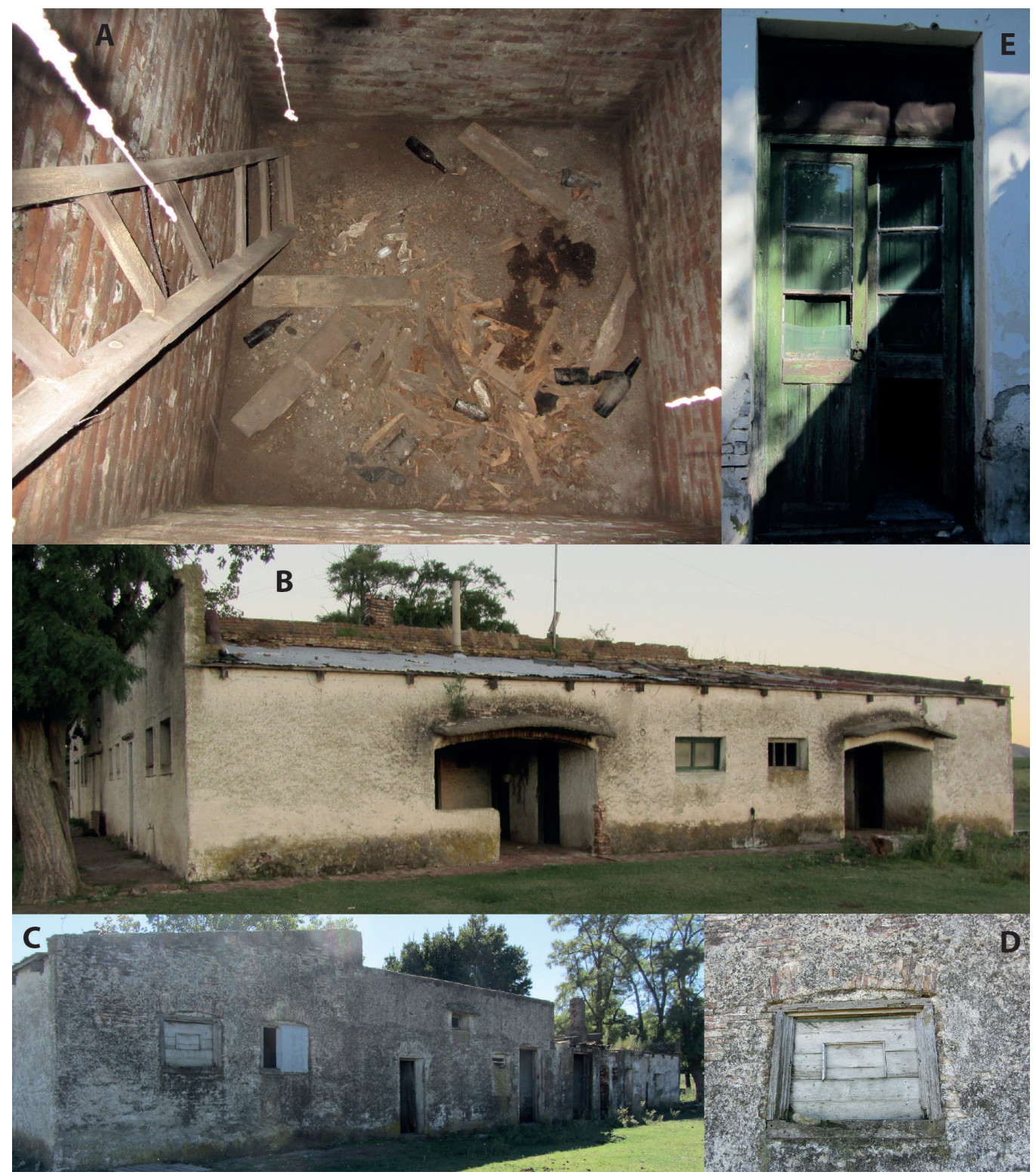

Figura 5. Detalles de San Carlos. A) Sótano con paredes de ladrillo, restos de escalera de madera. B) Lateral oeste de la construcción, detalle de teja y guardapolvos sobre las entradas. C) Contrafrente, se observan los dinteles rectos y las aberturas con sus guardapolvos. D) Detalle de ventana. E) Puerta de dos hojas con paños vidriados y banderola superior. 
este se observa una puerta de madera con una ventanilla (que conecta con el lateral este), al norte (comunicando al frente) una puerta de metal con cuatro paños de vidrio; hacia el oeste del patio dos habitaciones poseen puertas similares en madera, de dos hojas con tablero y con banderola superior vidriada, tres paños de vidrio en cada hoja y postigos internos (Figura $5 \mathrm{E}$ ). Tiene forma cuadrangular y mide $7 \mathrm{~m} \mathrm{x}$ $8,5 \mathrm{~m}$, posee una vereda de ladrillo, en el centro piso de tierra donde crece un árbol. La fachada del patio no presenta elementos dominantes, no subordinan las aberturas a ningún eje de simetría o motivo que posibilite una composición ritmada (según clasificación de Nadal Mora 1946).

El sector del lateral oeste tiene un estilo colonial con detalle de teja (Figura 5 B). Esto podría aportar un elemento clave a la hipótesis que el núcleo original del sitio sería el patio con las habitaciones conectadas al mismo, y que el lateral oeste sería una adición posterior, correspondiente al estilo neocolonial que se desarrolló a principios del siglo XX (Moreno 1996). Presenta en sus habitaciones piso de ladrillo y cielorraso de madera. Las habitaciones tienen pintura sobre el revoque y algunas de ellas poseen tragaluz. Los muros exteriores presentan revoque con granulado de conchilla. Las ventanas son apaisadas con una contraventana de madera que cierra la abertura y las puertas son de madera con mirilla. Desde el exterior se observan dos amplias entradas con dinteles de ladrillo en arco sobre los cuales hay guardapolvos, cornisa o alero, y entre ambas entradas hay dos ventanas rectangulares, una dividida en tres partes y otra en dos; pero cada entrada corresponde a un pasillo o patio cubierto que conecta varias habitaciones (Figura 5 B). De norte a sur, la primera entrada comunica cinco puertas de madera (que dan cada una a una habitación) que se caracterizan por poseer todas una ventanilla rectangular ( $\sin$ cerramiento en un caso, con un paño de vidrio en otra, y el resto cerradas con un postigo de madera), además se observan dos pequeñas ventanas cuadradas. La segunda entrada conecta tres habitaciones cuyas puertas de madera son similares a las anteriores. Según los relatos orales, habrian sido las habitaciones de los peones de la estancia (hombres solos). Actualmente este sector se utiliza como depósito.

En relación a las fases constructivas o a los distintos momentos en la vida del edificio, resulta dificil la determinación por las numerosas refacciones que se realizaron en el establecimiento, pero es posible definir al menos tres. Hasta el momento, no fue posible identificar qué sectores corresponden a la ocupación inicial, pero si algunos elementos permiten identificar modificaciones posteriores como ventanas y puertas tapiadas y paredes demolidas para unir espacios. También se aprecia un último período en la ocupación del sitio (estuvo habitado hasta hace pocos años atrás, y algunos espacios incluso son usados para guardar cosas en la actualidad) evidenciado por habitaciones que conservan pintura en sus paredes, utilizan materiales más nuevos como placas de yeso e incluso mantienen elementos sanitarios en funcionamiento.

\section{Caso 2: la casa del intendente (partido de Puan)}

El territorio ocupado actualmente por este Partido estuvo bajo dominio indígena y era asiento de tolderias en el siglo XIX. En 1875 con el avance general de las fronteras sobre la pampa, correspondió a la división de la Frontera Costa Sur la ocupación de los campos al oeste de Carhué hasta las tierras denominadas con la voz indigena "Puan" y la radicación de la comandancia de dicha línea. El arribo de la tropa, construcción de las dependencias 
militares y públicas y la correspondiente fortificación, se produjo entre los años 1876 y 1877 . Posteriormente se formó un pequeño conglomerado urbano con la llegada de familiares de la tropa y algunos comerciantes. La plaza fue la comandancia $\mathrm{y}$, con las 8 manzanas que la circundan, constituyen la zona fundacional. A cinco cuadras del centro, rumbo hacia la laguna se encuentra la plaza de la patria, que en época de la Campaña al Desierto se presume fue Cementerio Militar.

Dependió en un principio de la Jefatura de Policía de Fronteras de Guamini y luego del Juzgado de Paz de este distrito. En 1886 se promulgó la ley $\mathrm{N}^{\circ} 1827$ por la que se crea el partido de Puan y en 1887 el Poder Ejecutivo decreta la fundación del pueblo. En junio de 1886 se le asignan autoridades propias, entre las cuáles Rómulo Franco aparece mencionado en las fuentes como primer intendente debido a las gestiones realizadas para crear el partido. En 1890 gana las elecciones, pero es afectado por los acontecimientos revolucionarios de ese año, y las disputas entre conservadores y radicales, y en este marco, Franco y su gente se acantonan en la isla y dirimen sus diferencias a los tiros (Michelutti 2009). A partir de ahí, Franco es descripto como un dictador y surgen versiones que lo presentan como proscripto pasando temporadas en Uruguay y Paraguay. Según la documentación analizada, Franco solicita la escrituración de la isla en diciembre de 1889, alegando que la tiene poblada y cultivada hace varios años y declarándose antiguo poblador (expediente $\mathrm{n}^{\circ} 19399$ folio 654 de la Escribanía Mayor de Gobierno, Archivo Histórico de la Provincia de Buenos Aires).

Posteriormente, la residencia en la isla fue habitada por la familia Vallejo, algunos dicen entre 1917 y 1921, y otros hasta el año 1924, luego se instalaron Julián e Indalecio Dupouy y habilitaron las instalaciones para hospedaje, adonde llegaban clientes o amigos de residentes locales des- de Buenos Aires para pasar algunos días. Todavía en la isla había unas caballerizas que habian pertenecido a Franco, y las dos garitas construidas en piedra frente a la edificación. La gente del pueblo solía ir a pasar el día los domingos o feriados. En 1927, el señor Merienne adquiere la isla y destruye los pisos de la residencia de Franco en busca de sus armas (Michelutti 2009). Los siguientes años, otros vecinos de la localidad, también han buscado este tesoro en diferentes oportunidades, sin éxito. La casa deja de estar habitada a fines de la década de 1940, sus últimos ocupantes fueron los miembros de la familia Francisco (aproximadamente 20 personas), quienes vivieron desde la década de 1930 hasta el año 1948. Por último, hubo una propuesta de recuperarla en la década de 1960 y convertirla en un local nocturno, pero esta intención no prosperó finalmente.

\section{La casa de piedra en el siglo XIX}

En la década de 1880 el señor Rómulo Franco compró la isla donde edificó una casa en el extremo noroeste. En el folio 99 de la mensura $n^{\circ} 30$ del partido de Puan, correspondiente al ejido de la localidad de Puan efectuado por el ingeniero Rodolfo Moreno en el año 1888, aparece la isla como propiedad a nombre de Rómulo Franco (Figura 6A). Desde 1886 hasta 1890, fue designado primera autoridad (intendente) del partido de Puan, ejerciendo esta función intermitentemente. Vivió en la casa que construyó en la isla, cuyos restos aún se observan en la actualidad. Era una casa confortable, que contaba con los lujos de la época (hasta agua caliente), donde realizó fiestas y recepciones (Michelutti 2009). Los relatos orales de los pobladores más antiguos de la localidad de Puan refieren que la casona que Rómulo Franco poseía en la isla tenía alfombras y cortinas de terciopelo, y allí se hacian fiestas fastuosas (Miche- 
lutti 2009), y representan a este personaje como una figura de buen vivir. La diversidad identificada en las cerámicas históricas recuperadas durante las tareas de campo se corresponde con esta interpretación (Panizza y Devoto 2018). Otros relatos orales cuentan sobre posibles entierros de armas $u$ otros elementos pertenecientes a la época de Rómulo Franco, vinculados a las turbulencias políticas de la época.

\section{La casa de piedra en la actualidad}

Los restos relevados en la actualidad corresponden a una estructura rectangular de una planta, de aproximadamente 16 metros de ancho y 20 metros de largo, que no presenta techo y está en gran parte derrumbada, con sectores donde se observan materiales agregados en una época posterior a su construcción original. Estos materiales más modernos (bloques de hormigón y ladrillos huecos) corresponden a la remodelación iniciada en la década de 1960 que nunca se completó. Las paredes están construidas utilizando piedras típicas de la zona y ladrillos. Posee aberturas rectangulares, las ventanas están enrejadas y las puertas conservan restos de una estructura metálica que debe haber llevado vitraux en su época. De las estructuras anexas (caballerizas, torreones, tanques, entre otros), se relevaron los cimientos de los dos torreones en la playa, delante y a los costados de la casa principal; un pequeño pozo a aproximadamente 19 metros hacia el oeste de la casa, y un gran tanque multiuso confeccionado en piedra local ubicado en el cerrito cercano, a 300 metros. No pudieron registrarse los cimientos de las caballerizas por encontrarse bajo el agua, y hasta el momento no se han hallado evidencias asociadas al muelle que se utilizaba en esa época (al sur-suroeste de la construcción) ni del molino de madera que varios testigos aseguran que existió.

\section{Análisis formal del sitio}

La estructura fue edificada completamente con muros de piedras y ladrillos fácilmente distinguibles ya que en la actualidad el revoque cubre escasos sectores de los muros interiores. El techo en su mayor parte se ha derrumbado y sólo se conserva en el sector del pasillo y del piso no quedan evidencias porque fue levantado cuando se produjeron las remodelaciones. Los muros poseen dinteles de ladrillo en arco para conformar las puertas y ventanas y una cornisa ornamental en los mismos (Figura 6 B y C).

En su parte exterior presentaba 12 aberturas correspondientes a tres puertas y nueve ventanas, que se disponian de la siguiente forma: en la pared noreste tres ventanas orientadas a la laguna, en la pared sudeste dos ventanas, una puerta de metal vidriada de dos hojas con banderola superior vidriada, y una ventana; en el muro sudoeste solamente una puerta, y en la pared noroeste una ventana, una puerta igual a la descripta en primer lugar y dos ventanas más. Todas las ventanas eran rectangulares con dinteles de ladri1lo en arco y rejas españolas empotradas que presentaban una guarda superior y una inferior con motivos de rizos (Moreno 1995).

Internamente la casa se organizaba en nueve espacios (Figura 4): el pasillo principal de $18 \mathrm{~m}$ de largo x 2,8 m de ancho, que comunicaba al exterior mediante dos puertas, una en cada extremo, y conectaba con cuatro habitaciones, al noreste la sala y al sudoeste con un dormitorio, la cocina (que también tenía conexión con el exterior y estaba surtida de agua caliente por el tanque) y el comedor. Por la sala se tenía acceso hacia el noroeste al baño (revestido con finos azulejos y con accesorios de categoria) y a un dormitorio, y hacia el sudeste a otros dos dormitorios. En este caso no se observa ningún espacio abierto, uno de co- 


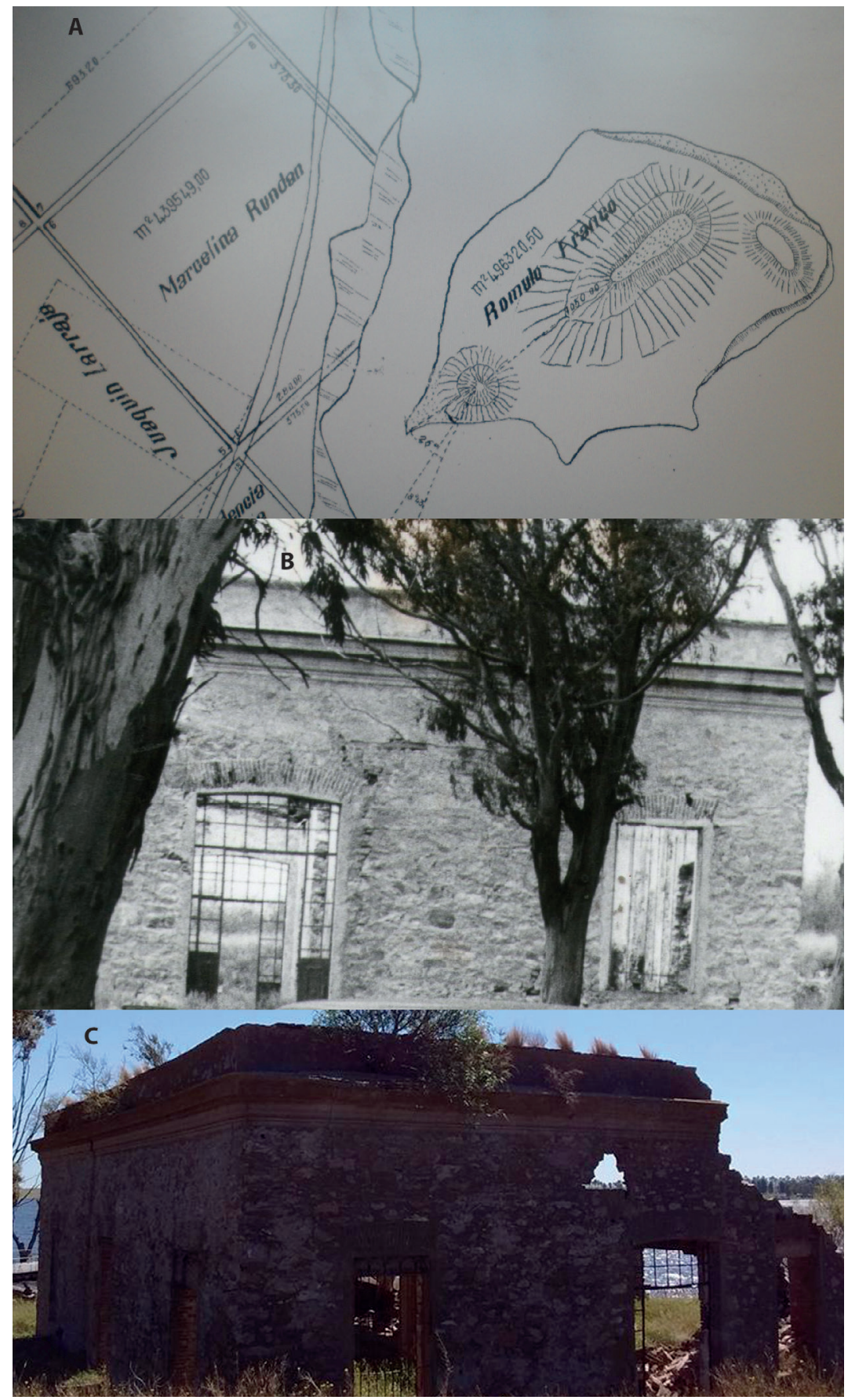

Figura 6. A) Detalle de la mensura $\mathrm{n}^{\circ} 30$ del partido de Puan (folio 99), año 1888, donde se observa la isla a nombre de Rómulo Franco. B) Detalles de la casa del intendente de Puan. Foto histórica donde se observa la cornisa de la construcción, las puertas de hierro de la entrada de la galería y una ventana enrejada, con sus dinteles en arco (proporcionada por Jorgelina Walter, Museo Ignacio Balvidares de Puan). C) Foto actual de la casa de piedra en ruinas. 
Figura 7. Estructuras anexas de la casa del intendente de la Isla de Puan. A) Fotografia histórica de los miradores o torreones (proporcionada por Jorgelina Walter, Museo Ignacio Balvidares de Puan). B) Fotografia de los cimientos de los miradores. C) Fotografia actual del gran tanque de agua del cerrito.

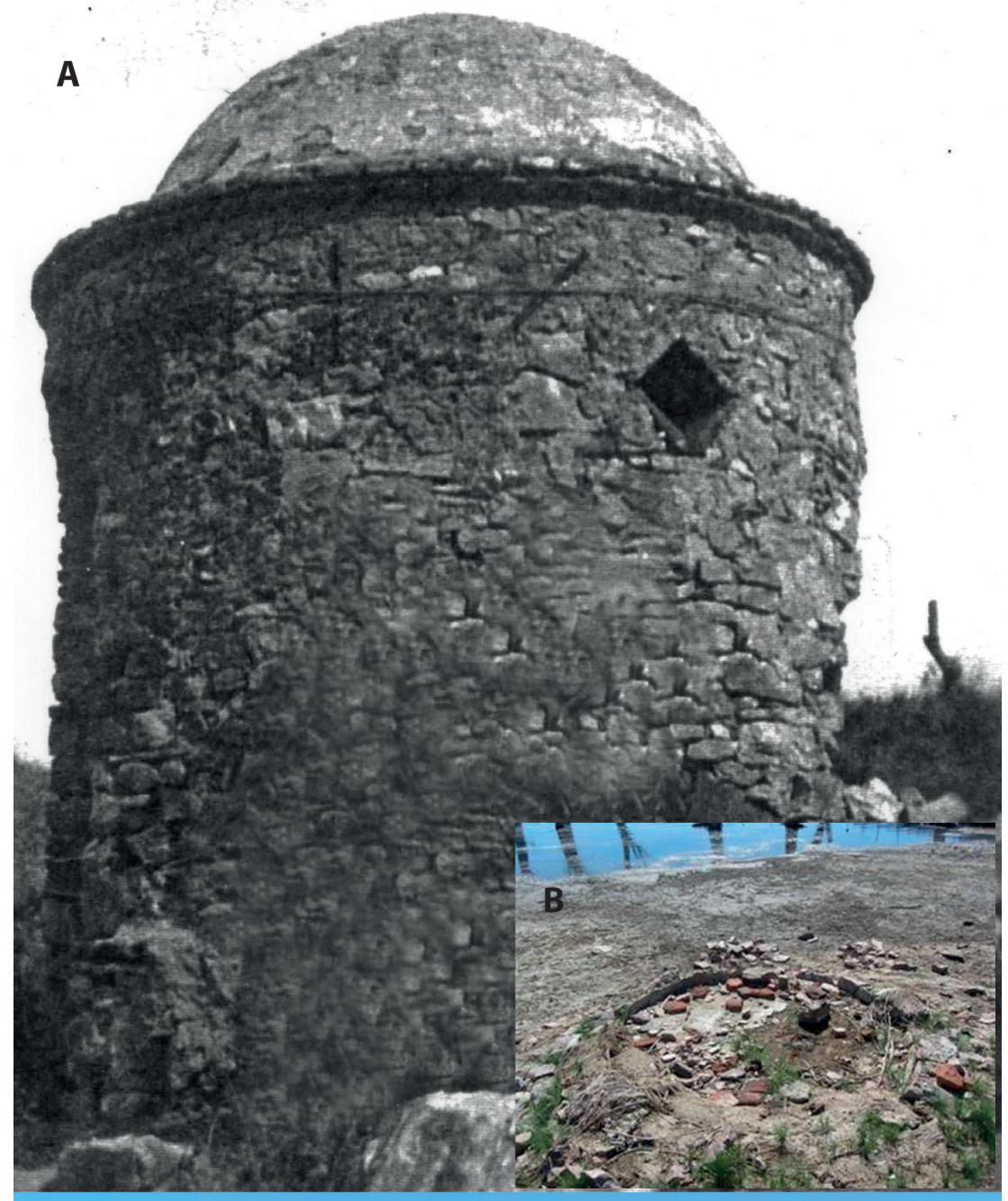

C

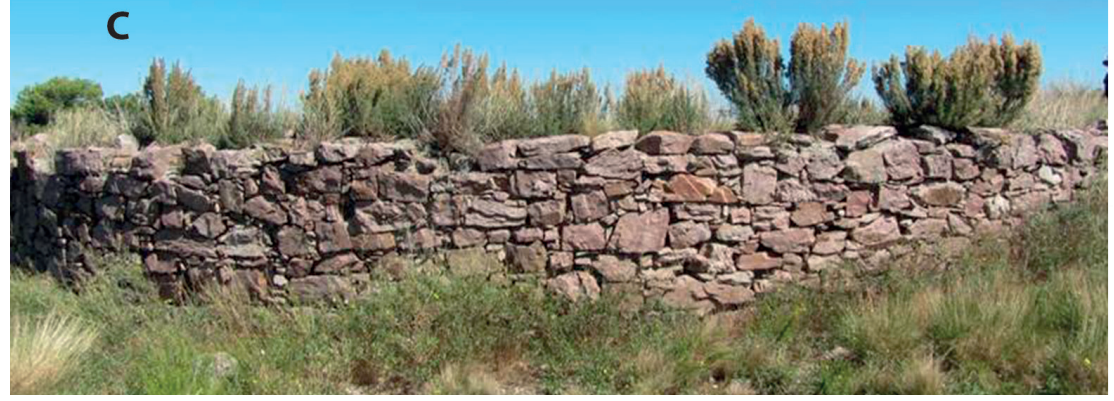


municación/circulación y ocho cerrados/ construidos.

Como edificios anexos a la residencia principal, funcionaban una caballerizas confeccionadas en piedra y ladrillo (actualmente sus cimientos se encuentran bajo agua) y dos miradores o garitas circulares elaborados con roca cuarcita local y techo cupuliforme, en los muros poseían unas pequeñas aberturas romboidales y en su interior una escalera de madera (Figura 7 A). Los miradores no subsisten en pie, pero sus cimientos pueden observarse en la playa junto al pelo de agua (Figura 7 B). También se refiere la existencia de un pozo de agua, el cual fue ubicado a $20 \mathrm{~m}$ aproximadamente durante las tareas de prospección del terreno. Por otra parte, a 300 $\mathrm{m}$ al oeste de la casa, en la cima de uno de los cerritos próximo se encuentra un gran tanque de agua (Figura $7 \mathrm{C}$ ), con paredes confeccionadas en la roca local (cuarcita rosada), al igual que la casa.

Con respecto a las fases constructivas de la edificación, es posible evidenciar con claridad al menos dos (el sitio tuvo variadas ocupaciones mencionadas en los relatos orales pero sólo se tendrá en cuenta en este aspecto la evidencia arqueológica y las modificaciones arquitectónicas observadas). De forma similar a lo descripto para el sitio San Carlos, no fue posible identificar qué sectores corresponden a la ocupación inicial, pero si se distinguen modificaciones posteriores como una ventana tapiada y aberturas en el sector posterior de la casa que en el plano original no aparecían. También se observan los cimientos de una construcción posterior en ese sector que habria sido realizada en el período que se quería remodelar para funcionar como un local nocturno, destinado a un jardín de invierno (Néstor Bucetta, comunicación personal). El mismo informante menciona que el sector de bloques de hormigón era el destinado para la cabina del disc-jockey, el sector de piso de cemento iban a ser los baños, y que hicieron la loza en el techo de la galería (hoy derrumbada, donde se observan ladrillos huecos y vigas de metal). Además se habrian quitado paredes divisorias internas en esta remodelación de la década de 1960.

\section{Consideraciones Finales}

Particularmente en el área de Ventania, se contaba con recursos de las sierras y las lagunas, por lo que gran parte de los materiales constructivos se aprovisionaron (piedra, tierra, arena) y fabricaron localmente (ladrillos). Los sitios analizados difieren en los recursos utilizados para la construcción de sus muros, ya que en el sitio Casa del Intendente se utilizó en gran medida la piedra por la disponibilidad de la misma en la isla de Puan y en menor medida el ladrillo, y en el sitio San Carlos se utilizó ladrillo fabricado localmente en la totalidad de la edificación. Ambas estructuras fueron revocadas y utilizaron en sus muros dinteles (en ambos sitios) y arcos (en el sitio San Carlos) para conformar las puertas y ventanas. Además las entradas de fachada están abiertas en el paramento mismo de ambos edificios. Ninguna de las construcciones presenta la galeria, elemento típico de la casa rural colonial y poscolonial (Nadal Mora 1946). Con respecto a la diversidad de puertas, en San Carlos se determinaron tres tipos (enteras, con ventanilla -abiertas en la hoja, hueco con postigos y/o con rejas - y vidrieras), en cambio en la casa de la isla del intendente sólo se identificó un tipo (vidriera).

Los momentos constructivos en la "vida" de los sitios fueron dificiles de determinar en ambos casos (independientemente de lo expresado por los relatos orales sobre la historia y las transformaciones de los sitios). En el sitio San Carlos (se identificaron por lo menos tres) por las numerosas refacciones realizadas a lo largo del siglo y porque se siguió utilizando hasta la actua- 
lidad y en el sitio Casa de Intendente (se identificaron por lo menos dos) porque está en gran medida demolido. En este sentido, es posible encontrar en ambos ventanas o puertas tapiadas, acciones que buscaban re-funcionalizar los espacios.

Los dos casos abordados en el presente trabajo consisten en construcciones habitacionales de una sola planta. Sin embargo, con respecto a los diferentes niveles de altura y circulación vertical en las dos estructuras, se observa que ambas presentaban acceso al techo y sótano. En el caso del nivel superior estariamos refiriéndonos a azoteas $^{5}$, desde donde se podía observar los alrededores y donde se ubicaban los tanques de agua. Debe recordarse que estas construcciones de azotea implican que el techo perdió protagonismo desde el punto de vista arquitectónico, y en general fueron acompañadas por un pretil ${ }^{6}$ de mampostería como forma de remate (Moreno 1996), tal como se observa en los dos sitios analizados. En San Carlos hay un sector de loza y otra de chapa, y en este nivel se ubica la campana sobre el frente. En la casa de Rómulo Franco de puerta a puerta había loza en el nivel superior (actualmente todo ese nivel no se observa porque se derrumbó/ ha desaparecido), y los relatos orales refieren que desde ahí se tiroteaban durante la primera etapa de ocupación de la edificación. Además el tanque de agua de agua fría y caliente que surtía a la casa estaba hecho en piedra con revoque, y quedaba oculto por la marquesina. Las dos edificaciones contaban con un nivel subterráneo, en el caso de San Carlos se descubrió un sótano bajo un piso de madera durante el relevamiento de la estructura edilicia, el cual tenía las paredes de ladrillos, piso de tierra, una escalera de madera rota y restos de basura como botellas (Figura 5 A). En cambio, en la casa de piedra de la isla de Puan habrían existido dos sótanos con paredes de tierra, los cuales habrian sido definitivamente tapados durante las remodelaciones de la década de 1960.

Por otra parte, las dos edificaciones están relacionadas con otras estructuras anexas, por lo cual en ambos sitios se pueden analizar las relaciones con las estructuras externas y circundantes a la construcción principal por una parte, y las vinculaciones con los espacios al interior de la estructura central. A esto se puede referir como el afuera y el adentro de los sitios.

Los sitios representan dos escalas y esferas de acción distintas. La casa del primer intendente de Puan es un espacio residencial y familiar con una pequeña unidad productiva constituida por los frutales, el tanque de agua, pequeño sector de cultivo, las caballerizas y cría de ganado. En una escala mucho más amplia y de formación más heterogénea del grupo residencial, se encuentra San Carlos, con actividades productivas a gran escala, vinculadas con actividades agrícola-ganaderas.

Con respecto a las sucesivas generaciones que habitaron estos espacios residenciales, en el caso de San Carlos tuvo dos propietarios, el matrimonio Cadmus a fines del siglo XIX y la familia Videla Dorna Serigós desde principios del siglo XX hasta ahora, pero estuvo habitada general e ininterrumpidamente por los trabajadores del campo, ya que los dueños preferentemente vivieron en la casona de estilo inglés, al otro lado del arroyo El Perdido. Dentro de la edificación, había una jerarquización de los espacios según quienes eran los que los habitaban, representada a través de los tipos de revestimientos, pisos, cielorrasos y otros tipos de enseres (como los artefactos de baño): las habitaciones del frente ocupadas por los administradores y sus familias, las habitaciones del lateral oeste (aisladas en cuanto a circulación del patio central y estructura principal) por peones $\mathrm{u}$ otros trabajadores masculinos solteros; y por último los espacios comunes, de tra- 
bajo y de depósito. En cambio, la casa de piedra de la isla de Puan experimentó el traspaso a través de sucesivas manos (Rómulo Franco, Vallejo, Meriennne, Francisco), luego estuvo deshabitada aproximadamente 20 años entre las décadas de 1940 y 1960, hubo un intento de remodelación y luego el abandono definitivo, con el consiguiente derrumbe de la mayor parte de la estructura.

El estado de conservación de ambas estructuras es diferente. El sitio San Carlos mantiene en pie todos los techos y muros externos, sin embargo algunos muros internos habrian sido demolidos y algunas habitaciones no conservan el piso. Actualmente es posible hallar en algunas habitaciones pinturas en las paredes, azulejos, placas de yeso y grifería. Por otro lado, la casa del primer intendente está en ruinas, los pisos no se conservan, el techo sólo en algunos sectores y quedan en pie solamente los muros internos correspondientes al pasillo, los del resto de las habitaciones no se conservan. Los muros externos pueden ser observados en la actualidad e incluso las rejas de algunas aberturas.

Por último, pueden mencionarse algunas evidencias que podrian funcionar como indicadores cronológicos de tipo indirecto. En primer lugar, con respecto a las cubiertas de las construcciones, debe considerarse que las láminas de hojalata ondulada o chapas de zinc se impusieron en Buenos Aires a partir de 1838-1840 (Schavelzon 1991), y a comienzos del siglo XX teja española moderna (hacia 1900), y los techos de azotea se generalizaron desde fines del siglo XVIII y a través del siglo XIX. Ambos sitios presentan ladrillos como material constructivo, elaborados localmente, y responden a los parámetros esperables para aproximadamente 1880: 15 x 30 x $5,5 \mathrm{~cm}$ (Moreno 1995). Además comparten la presencia de dinteles en arco de ladrillo para los vanos de las aberturas (puertas y ventanas). En cuanto a las aberturas, tam- bién aportan información importante, algunas de las puertas de madera con paños vidriados corresponden morfológicamente a aquellas observadas en otras residencias de las décadas de 1860 y 1870, y las que presentan banderola a la década de 1890 (Moreno 1995).

A partir de este trabajo se procuró aplicar una estrategia de investigación que estudia la arquitectura de los sitios históricos con estructuras remanentes mediante una metodología arqueológica cuyo principal objetivo es el conocimiento histórico de los contextos construidos y de las sociedades que los generaron, y que los resultados obtenidos puedan contribuir a su gestión integral. La importancia de promover su conservación radica en el hecho que un edificio histórico es un documento que aporta información sobre sociedades pasadas (Azkarate 2013), y como forma parte de la cultura material puede ser estudiado con una metodología arqueológica. En este sentido, se espera aportar a la elaboración de una historia social del pasado a través del registro arqueológico (Azkarate et al. 2002). La información que proporciona la lectura arqueológica de las edificaciones puede servir como un instrumento para proyectar su conservación. El uso actual que se otorgue a este tipo de patrimonio dependerá de muchos factores, tales como politicas patrimoniales, su estado de preservación y su reconocimiento social (Blanco Rotea 2017). Por otra parte, se considera que una aproximación simbiótica a la arquitectura y el paisaje resulta operativa, ya que ambos conceptos suponen la manifestación de códigos sociales de un determinado grupo humano a través del paisaje que construye y cómo lo construye.

El estudio de las relaciones espaciales entre los diversos elementos de una construcción, es decir su estructura espacial, permite un acercamiento a la lógica espacial de una determinada comunidad. Los análisis formales que fueron realizados en 
ambos sitios contribuyeron a la compresión del conjunto de relaciones inter-espaciales que construyó la sociedad de fines del siglo XIX en el período final de frontera (sitio San Carlos) y comienzos de la etapa constitucional de los pueblos de Ventania (sitio Casa del Intendente).

Se destaca la importancia de complementar el análisis formal realizado sobre la arquitectura de los sitios arqueológicos con las fuentes documentales disponibles, con el análisis de los restos materiales hallados en los sitios y con la memoria oral de las comunidades locales para alcanzar una comprensión integral sobre de la construcción social del espacio de un determinado grupo.

\section{Agradecimientos}

A Rodolfo Serigós y familia, de la estancia Santa Ana en el partido de Coronel Suárez. A la gente del Municipio de Puan que colaboraron en las actividades; Facundo Castelli, el Intendente; Cora Biondini, la Directora de Turismo; especialmente a Jorgelina Walter, la Directora del Museo Ignacio Balvidares; Cristina Bartolomé, José Luis Strack, el encargado de la Isla; la familia Francisco, Roberto Héctor Rueda y Néstor Bucetta. A Lucas Martínez y Anabella Sfeir, que participaron en las tareas de campo en Coronel Suárez. Este trabajo se enmarcó en el proyecto de investigación UNR HUM 525, una Beca Post-Doctoral CONICET y una Beca Doctoral UNLP.

\section{Notas}

1. El brocal es el antepecho alrededor de la boca de un pozo.

2. El contrafuerte es la parte de una obra que sobresale del paramento de un muro a manera de macizo vertical, que sirve de apoyo o refuerzo y que neutraliza los empujes trasversales.

3. Guardapolvos es un término del campo de la arquitectura, con el cual se designa a un pequeño tejado en voladizo, construido sobre balcones, puertas y ventanas, con el objeto de protegerlos del agua de lluvia y del sol.

4. La espadaña es el campanario formado por una sola pared o muro perforado por un hueco en el que se aloja la campana.

5. La azotea es la cubierta de un edificio, plana o en ligero declive, por la cual se puede transitar.

6. El pretil alude a la barandilla o antepecho de piedra o ladrillo.

Todas estas definiciones fueron extraídas del Glosario de Arquitectura elaborado por el Instituto de Patrimonio Cultural, Quito, 2010 .

\section{Bibliografia}

AZKARATE GARAI-OLAUN, A.; L. CABALLERO ZOREDA y J.A. QUIRÓS CASTILLO. 2002. Arqueología de la Arquitectura: definición disciplinar y nuevas perspectivas. Arqueología de la Arquitectura 1:7-10.

http:/ / arqarqt.revistas.csic.es /index. php/arqarqt/article/view/1/1.

AZKARATE GARAI-OLAUN, A. 2013. La construcción y lo construido. Arqueología de la Arquitectura. En La materialidad de la historia. La arqueología en los inicios el siglo XXI, editado por J. Quirós Castillo, pp. 271-298. Akal, Madrid.

BALDRICH, A. 1896. En Curá-Malal. La División Buenos Aires. Primera Conscripción Argentina. La Harlem, Buenos Aires.

BERÓN, M. y E. I. BAFFI 2003. Procesos de cambio cultural en los cazadores-recolectores de la provincia de La Pampa, Argentina. Intersecciones en Antropologia 4:29-43,

BLANCO-ROTEA, R. 2017. Arquitectura y paisaje. Aproximaciones desde la arqueología. Arqueología de la Arquitectura 14:e051. http://dx.doi. org/10.3989/arq.arqt.2017.007.

CRIADO BOADO, F. 2012. Arqueológicas. La razón perdida. Ediciones Bellaterra, 
Barcelona.

CRIADO BOADO, F. y P. MAÑANA BORRAZÁS. 2003. Arquitectura como materialización de un concepto. La espacialidad Megalitica. Arqueología de la arquitectura 2:103-111.

DEVOTO, M.G.; M.C. PANIZZA y F. OLIVA. 2016. La frontera militar en el Sistema Serrano de Ventania a fines del siglo XIX. Revista TEFROS 14(2):63-86.

LANGIANO, M.C. y J.F. MERLO. 2013. Camino de los indios a Salinas. Arqueología y paisaje en la Frontera Sur (provincia de Buenos Aires 1850-1880). Anuario de Arqueología (UNR) 5:169-188.

LANGIANO, M.C.; J.F. MERLO y P. ORMAZABAL. 2002a. Relevamiento de Fuertes y Fortines, con relación al denominado camino de los indios a salinas. En Del Mar a los Salitrales Diez mil Años de Historia Pampeana en el Umbral del Tercer Milenio, editado por D.L. Mazzanti, M. Berón y F. Oliva, pp. 53-64. Laboratorio de Arqueologia, Facultad de Humanidades, Universidad Nacional de Mar del Plata, Mar del Plata.

LANGIANO, M.C.; J.F. MERLO y P. ORMAZABAL. 2002b. Modificaciones en el basural del Fuerte San Martín. En Arqueología Histórica Argentina. Actas del primer Congreso Nacional de Arqueología Histórica, pp. 389-393. Ediciones Corregidor, Buenos Aires.

MANNONI T., 1990. Archeologia dell'architettura. Notiziario di Archeologia Medievale 54:28-29.

MAÑANA BORRAZÁS, P. 2003. Arquitectura como percepción. Arqueología de la arquitectura 2:177-183.

MAÑANA BORRAZÁS, P.; R. BLANCO ROTEA y X.M. AYÁN VILA. 2002. Bases teórico- metodológicas para la práctica de la Arqueología de la Arquitectura. TAPA. Traballos de Arqueoloxía e Patrimonio 25:12-101.

MERLO, J.F. 2007. Avances sobre el uso de recursos faunísticos en la dieta de los habitantes de puestos fortificados en el Camino a Salinas (área Interserrana Bonaerense). Intersecciones en Antropología 8:185-196.

MICHELUTTI, C. 2009. Cronología para la Historia de Puan III. Editorial Dunken, Buenos Aires.

MONFERRAN MONFERRAN, E.E. 1958. Batalla de Currumalan-Chico. 20 de abril de 1877. Batalla de las Sierras de Pigüé. SUPRINT, Buenos Aires.

MONFERRAN MONFERRAN, E.E. 1962. El ejército de operaciones del Sud y la batalla del Pihué. Imprenta del Congreso de la Nación, Buenos Aires.

MORENO, C. 1995. De las viejas tapias y ladrillos. ICOMOS Comité Argentino, Buenos Aires.

MORENO, C. 1996. Yendo, viniendo y poblando. ICOMOS Comité Argentino, Buenos Aires.

NADAL MORA, V. 1946. Estética de la arquitectura colonial y postcolonial argentina. El Ateneo, Buenos Aires.

OLIVA, F. 2006. Uso y contextos de producción de elementos "simbólicos" del sur y oeste de la pcia. de Bs. As., República Argentina (área Ecotonal Húmeda Seca Pampeana). Revista de la Escuela de Antropología (UNR) XII:101-115.

OLIVA, F.; M. ALGRAIN y M.C. PANIZZA. 2015a. Investigación-Extensión en la Arqueología del Área Ecotonal Húmeda Seca Pampeana (AEHSP). Abordajes desde el Centro de Estudios Arqueológicos Regionales (CEAR). Anuario de Arqueologia (UNR) 7:131-145.

OLIVA, F.; L. L'HEUREUX; H. DE ANGELIS; V. PARMIGIANI y F. REYES. 2007. Poblaciones indigenas de momentos postcontacto en el borde occidental de la pampa húmeda: Gascón 1, un sitio de entierros humanos. En Arqueología Argentina en los inicios de un nuevo siglo. Tomo I, , editado por F. Oliva, N. De Grandis y J. Rodriguez, pp. 265-274. Laborde, Rosario. 
OLIVA, F. y M.L. LISBOA. 2006. El estudio de cuentas en diferentes contextos arqueológicos del Sistema de Ventania y su llanura Adyacente. Revista de la Escuela de Antropología (UNR) XII:135148.

OLIVA, F. y M.L. LISBOA. 2009. Indicadores arqueológicos de cambio cultual en las comunidades indigenas pampeanas de los primeros momentos históricos (siglos XVI a XVIII). Región Pampeana, República Argentina. En Arqueología Colonial Latinoamericana. Modelos de estudio, editado por J. García Targa y P. Fournier García, pp. 255-267. BAR Internacional Series 1988, Archaeopress, Oxford.

OLIVA, F. y M.C. PANIZZA. 2015a. Investigaciones Arqueológicas en el Área Ecotonal Húmeda Seca Pampeana. En Ciencia y tecnología 2015: divulgación de la producción científica y tecnológica de la UNR, editado por C. López, pp. 10771083. UNR Editora, Rosario.

OLIVA, F. y M.C. PANIZZA. 2015b. Evidencias de contacto hispano indígena a través del estudio de las representaciones rupestres de las sociedades indígenas tardias del Sistema Serrano de Ventania (Provincia de Buenos Aires, República Argentina). En Arqueología y Etnohistoria Del Centro-Oeste Argentino, Publicación de las X Jornadas de Investigadores en Arqueologia y Etnohistoria del Centro-Oeste del País, editado por A.M. Rocchietti, A. Austral, G. Pérez Zavala, R. Nuñez Ozan y D. Reinoso,pp. 44-67. UniRío Editora, Río Cuarto, Córdoba.

OLIVA, F. y M.C. PANIZZA. 2016. La representación de una "embarcación" como evidencia del contacto hispano indigena en el Sistema Serrano de Ventania (Provincia de Buenos Aires, República Argentina). Revista de la Escuela de Antropologia (UNR) XXII:231-253.

OLIVA, F. y M.C. PANIZZA. 2017. Análisis de la cerámica arqueológica del Área Ecotonal Húmedo Seca Pampeana. En Investigaciones arqueométricas: técnicas $y$ procesos, editado por A.M. Rocchietti, F. Ribero y D. Reinoso, pp. 105-120. Aspha Ediciones, Buenos Aires.

OLIVA, F. y M.C. PANIZZA. 2018. Arqueología histórica en el Área del Sistema Serrano de Ventania y su llanura adyacente. En Ciencia y Tecnología 2017: divulgación de la producción cientfica y tecnológica de la UNR, editado por E. Orellano, L. Bulacio, C. Pairoba, P. Ponce de León y J. Molero, pp. 1335-1343. Editorial de la Universidad Nacional de Rosario, Rosario.

OLIVA, F.; M.C. PANIZZA; L. CATELLA; J. MOIRANO; N. MORALES; M. ALGRAIN; G. DEVOTO; L. IANNELLI; C. OLIVA; B. PEREYRA y A. SFEIR. 2015b. La construcción del pasado arqueológico en diferentes sectores del Ârea Ecotonal Húmedo-Seca Pampeana. Investigación y Extensión desde el Centro de Estudios Arqueológicos Regionales. Revista de Antropología del Museo de Entre Ríos 1(2):91-102.

OLIVA, F.; M.C. PANIZZA y G. DEVOTO. 2015c. El sector sur del Área Ecotonal Húmedo Seca Pampeana en el siglo XVIII. El sitio Laguna Gascón 1 y sus materiales metálicos. Revista Teoría y práctica de la Arqueología Histórica Latinoamericana IV(4):147-161.

OLIVA, F.; M.C. PANIZZA y M.G. DEVOTO 2016. Aproximación a la frontera en el sector sur del Área Ecotonal Húmedo Seca Pampeana: investigaciones en el sitio San Carlos (partido de Coronel Suárez, pcia. de Buenos Aires). Anuario de Arqueología 8:217-236.

PANIZZA, M.C. 2015. El área de Ventania en la conformación de la línea de frontera durante el siglo XIX. Cambio y diversidad cultural en la apropiación del paisaje.Revista TEFROS 13(2)83-107.

PANIZZA, M.C. y M.G. DEVOTO. 2018. 
Arqueología histórica en el partido de Puan (provincia de Buenos Aires). Revista de Antropología del Museo de Entre Ríos 4(1):87-101.

PANIZZA, M.C.; F. OLIVA y M.G. DEVOTO. 2016. Investigaciones arqueológicas en la casa del primer intendente de la localidad de Puan, Provincia de Buenos Aires. Revista Teoría y Práctica de la Arqueología Histórica Latinoamericana $\mathrm{V}(5): 131-144$.

PETZ, R. y M. SAGHESSI. 2007. Estudio del material lítico del área de las Encadenadas, partido de Guaminí (Provincia de Buenos Aires). En Arqueología Argentina en los inicios de un nuevo siglo, tomo I, editado por F. Oliva, N. de Grandis y J. Rodríguez, pp. 615-620. Laborde, Rosario.

RAONE, J.M. 1969. Fortines del desierto (mojones de civilización). Tomos I, II y III. Biblioteca del Suboficial $n^{\circ} 143$, Buenos Aires.

ROA, M. y M. SAGHESSI. 1998. Investigaciones arqueológicas en el Fortín Recompensa. Partido de Guaminí. Pcia. de Bs. As. En Actas de las $I^{\circ}$ Jornadas Regionales de Historia y Arqueología del siglo XIX, editado por Programa de Investigaciones Antropológicas en Tapalqué (PIAT), pp. 89-93. PIAT e INCUAPA Publicaciones, Olavarría.

SCHAVELZON, D. 1991. Arqueología Histórica de Buenos Aires. La cultura material porteña de los siglos XVIII y XIX. Ediciones Corregidor, Buenos Aires.

SENNET, R. 1991. La conciencia del ojo. Versal, Barcelona.

THILL, J. y J. PUIGDOMENECH. 2003. Guardias, Fuertes y Fortines de la Frontera Sur. Historia, Antecedentes y Ubicación Catastral. Tomos I y II. Servicio Histórico del Ejército Argentino, Editorial Edivern, Buenos Aires.

UTRERO AGUDO, M.A. 2011. Archaeology. Archeologia. Arqueología. Hacia el Análisis de la Arquitectura. En Arqueología aplicada al estudio e interpretación de edificios históricos. Últimas tendencias metodológicas, editado por M. Domingo Fominaya y A.J. Sánchez Luengo pp. 11-23. Ministerio de Cultura, Madrid.

VIGIL, C. 1959. Los monumentos y lugares históricos de la Argentina. Editorial Atlántida, Buenos Aires.

VILA, M. 2013. Uso de la Piedra en la construcción de los Fortines del Desierto. http: / / www.slideshare.net/nmollo/ vila-m-2013-uso-de-la-piedra-en-laconstruccin-de-los-fortines-del-desierto 